\title{
Radioastron pulsar early science program: Current status and results
}

\author{
Andrey Andrianov ${ }^{1}$ \\ Astro Space Center, Lebedev Physical Inst. RAS, Profsoyuznaya 84/32, 117997 Moscow, Russia \\ E-mail: andrianeasc.rssi.ru
}

The Radioastron Early Science Program (ESP) is a plan of operations to achieve first scientific results of the RadioAstron (RA) Ground-Space VLBI mission. The preliminary goal of the ESP is to obtain high profile scientific results and to provide a connection between the start of the early Radioastron operations/observations/data processing and the future standard observations by international VLBI community users.

Pulsars offer a variety of effects caused by interstellar plasma. Dispersion by interstellar plasma allows the measurement of the electron column density along the line of sight and this provides an indication of the distance to the pulsar. Scattering effects include scintillations (variations of intensity with time), frequency distortion (decorrelation bandwidth) and angular broadening. These observable issues provide us an ability to study the scattering material as a large, defective lens, producing an image of the pulsar, convolved with the response to a point source at the observer plane.

The RA mission makes possible the detection of angular broadening for many pulsars. With RA it is possible to observe the nearby pulsars with the longest VLBI baselines, up to $300000 \mathrm{~km}$. We report some results of data processing for RA pulsar experiments. We present the first direct assessments of pulsar broadening, make some constraints on the size of the emitting region and evaluate the distribution of the scattering medium by studying the dynamic cross-spectra and fringes of the Earth-Space baselines. The pulsar mode of the ASL Correlator and its particular features are also discussed.

11th European VLBI Network Symposium \& Users Meeting

October 9-12, 2012

Bordeaux, France

\footnotetext{
Speaker

on behalf of the RadioAstron ESP Working Group
} 


\section{Introduction}

This presentation was prepared by the pulsar ESP working team which insludes M. Popov, C. Gwinn, A. Andrianov, N. Bartel, M. Johnson, E.C. Joshi, M. Kramer, V. Shishov, T. Smirnova, V. Soglasnov, V. Zhuravlev, and collaborators...

The RadioAstron project is an international collaborative mission which uses a free-flying satellite, Spektr-R, carrying a 10-m space radio telescope (SRT) on an elliptical orbit around the Earth. The aim of the mission is to use the space telescope for radio astronomical observations using VLBI (Very Long Baseline Interferometry) techniques in conjunction with ground-based VLBI networks. The orbit of the RadioAstron satellite evolves with time and has an apogee between 280,000 and $350,000 \mathrm{~km}$, a perigee between 7,000 and $80,000 \mathrm{~km}$, a period of 8 to 9 days, and an initial inclination of $51^{\circ}$. RadioAstron operates at the standard radio astronomical wavelengths of 1.19-1.63 cm (K-band), $6.2 \mathrm{~cm}$ (C-band), $18 \mathrm{~cm}$ (L-band), and $92 \mathrm{~cm}$ (P-band).

\subsection{Pulsars are among prospective objects for space VLBI}

Pulsars are very compact objects with angular sizes less then $1 \mu$ as, and they may be very conveniently studied with Space VLBI. It allows to investigate interstellar scattering and pulsar emission mechanisms, as described in 4 categories below.

\section{Local scattering material}

Local scattering material scatters both nearby pulsars and extragalactic intra-day variable sources. The angular broadening is too small to be detected with Earth-based baselines, but should be easily resolved with baselines to Radioastron. The local material appears to scatter in a fundamentally different way from more distant material, through refractive rather than difractive scattering [1]. The material that scatters IDV (intra-day variable) sources appears to be highly elongated $(10: 1)$ and extremely close $(\mathrm{d}<12 \mathrm{pc})$ [2]. Observations of nearby pulsars on a variety of baselines will test this picture. From the measured angular broadening and the characteristic bandwidth of scintillation, we can estimate the distance of the scattering material.

2. Substructures within the scattering disk

The source with large disperse measure values (DM values) is expected to be visible in the speckle limit, but with non-persistent fringes with completely random phase and amplitude variations over the scintillation bandwidth, on this long baseline. Narayan \& Goodman [4] proposed that the visibility might arise on long baselines, because of "fractal" substructures within the scattering disk. They suggest that such substructures would carry information about the fluctuations responsible for scattering, and might even allow interferometric imaging in spite of scattering.

\section{Resolving pulsar emitting regions}

Spatially resolving the source of pulsar emission requires nanoarcsecond resolution, and a lens with a diameter of an AU. Interstellar scattering provides such a lens, although it is highly corrupt. Nevertheless the statistics of the visibility provide information on the size, elongation, and position shift, and their variation over the pulse $[3,5,6]$

The figures of suitability for such observations include the size of the light cylinder (presumably related to the size of the emitting region) measured in units of the resolution of 
interstellar scattering viewed as a lens, observation of many scintillation elements within one recording bandwidth, and sufficient signal-to-noise ratio within one scintillation element so we can characterize the distribution.

\section{Giant pulses}

Pulses from all pulsars vary in intensity, but the flux density of the Crab is dominated by the strongest pulses. Strong pulses have durations of microseconds and vary on the Nyquist rate [7]. A single such giant pulse can have a flux density in excess of $10^{6} \mathrm{Jy}$ [8]. The short duration of such pulses indicates that they find their origin in an extremely compact region. The complicated structures hint that the propagation physics, such as multipath propagation, may be important. Scattering of the Crab pulsar is dominated by the surrounding Crab Nebula, as indicated by comparison of the angular and temporal broadening [9].

Radioastron observed giant pulses from the Crab as part of the Early Science Program. The best-observed giant pulse is clearly observable at Radioastron and at large telescopes on Earth. Interestingly, the shape of the pulse varies with station position. This suggests that the propagation in the Crab Nebula determines the shape of the giant pulse, since this spatial scale is farther than a beamed source within the light cylinder could produce, given the optics of wave difraction. The distribution function of the intensity does not match that of a scattered point source, but does match that of a resolved source, suggesting that the scattering in the Crab Nebula acts as a lens to spatially resolve the source.

\section{Observations}

Radioastron pulsar ESP group submitted several applications for obtaining observing time at ground-based telescopes: "Radioastron-HAS Observations of Nearby Pulsars" (Proposal ID: VLBA/12B-247), "Radioastron-LBA Observations of the Vela Pulsar" (Proposal ID:2012APRS/V479), "Radioastron-EVN Observations of Giant Pulses from the Crab Pulsar" (Proposal ID:E11C019), "Observations of Giant Pulses from the Crab and Milisecond Pulsars" (Proposal ID:E12B016). All of these applications have been approved and observed time was allocated in a full volume. Table 1 . below shows the status of all observing sessions.

\section{Data Correlation}

Pulsar experiments were processed on ASC correlator. ASC Correlator has several modes for pulsar processing:

- Simple Gate Mode (allows to increase $\mathrm{S} / \mathrm{N}$ ratio up to 3-5 times)

- Compound Gate Mode: the gate is weighted by the average profile (allows to increase $\mathrm{S} / \mathrm{N}$ ratio up to $6-20 \%$ relative to the Simple Gate mode)

- Bins Mode: many gates (Bins) are used when the on-pulse phase is unknown. The mean profile can be obtained with this mode. 
Table 1. Status of observing sessions

\begin{tabular}{|c|c|c|c|c|c|c|c|c|}
\hline$\#$ & RA_code & Other_code & Pulsar & Date & Time (h) & Range & Telescopes & Status \\
\hline 1 & Rafs01 & & \begin{tabular}{|l} 
B0531+21 \\
Crab
\end{tabular} & 15.11 .11 & 1 & $\mathbf{L}$ & Bd,Ev,Sv,Zk & Processing \\
\hline 2 & Rafs12 & & B0950+08 & 25.01 .12 & 1 & $\mathbf{P}$ & Ar,Ef,Wb & Processed \\
\hline 3 & Raes04a & Eg060 & \begin{tabular}{|l} 
B0531+21 \\
Crab GP
\end{tabular} & 02.03 .12 & 4 & $\mathbf{L}$ & $\begin{array}{l}\text { Bd,Ef,Ev,Hh,Jb, } \\
\text { On,Sv,Ur,Wb }\end{array}$ & Processing \\
\hline 4 & Raes04b & Eg060 & \begin{tabular}{|l} 
B0531+21 \\
Crab GP
\end{tabular} & 06.03 .12 & 3 & $\mathbf{L}$ & $\begin{array}{l}\text { Bd,Ef,Ev,Hh,Jb, } \\
\text { On,Sv,Ur,Wb }\end{array}$ & Processing \\
\hline 5 & Raes06a & V2692 & $B 0823+26$ & 18.04 .12 & 2 & $\overline{\mathbf{P}}$ & $\mathbf{A r}, \mathbf{E v}$ & Observed \\
\hline 6 & Raes06b & $\mathrm{V} 2692$ & B0950+08 & 19.04 .12 & 0.5 & $\mathbf{P}$ & Ar & Observed \\
\hline 7 & Raes06c & V2692 & B0834+06 & 26.04 .12 & 2 & $\mathbf{P}$ & Ar,Ef & Procesing \\
\hline 8 & Raes07a & & \begin{tabular}{|l} 
B0833-45 \\
Vela
\end{tabular} & 10.05 .12 & 4 & $\mathbf{L}$ & Нh,Мp,Рa,Тi & Processing \\
\hline 9 & Raes07b & & Vela & 18.05 .12 & 1.5 & $\mathrm{~L}$ & Hh,Мp,Pa,Ti & Processing \\
\hline 10 & Raes09 & & $B 1508+55$ & 07.06 .12 & 1.5 & $\mathbf{P}$ & Ev,ORT & Observed \\
\hline 11 & Raes06d & V2692 & B1919+21 & 04.07 .12 & 2.3 & $\mathbf{P}$ & Ar,Gb,Wb & Processed \\
\hline 12 & Raes06e & V2692 & $\mathrm{B} 1929+10$ & 07.08 .12 & 1.0 & $\mathbf{P}$ & & No ground \\
\hline 13 & Raes06e & V2692 & $B 2016+28$ & 08.08 .12 & 1.1 & $\mathbf{P}$ & & No ground \\
\hline 14 & Raes06e & $\mathrm{V} 2692$ & $\mathrm{~B} 1929+10$ & 01.09 .12 & 1.5 & $\mathbf{P}$ & Ar,Wb & No space \\
\hline 15 & Raes06f & $\mathrm{V} 2692$ & B0525+21 & 05.10 .12 & 2.0 & P & Ar,VLBA & scheduled \\
\hline 16 & Raes04c & EP067A & $\mathrm{B} 1937+21$ & 22.10 .12 & 3.0 & $\mathbf{L}$ & Ar,EVN & scheduled \\
\hline 17 & Raes04d & EP067B & Crab & 23.10 .12 & 2.0 & $\mathbf{L}$ & Ar,EVN & scheduled \\
\hline 18 & Raes06g & HSA12B-247 & B0329+54 & $26-29.11 .12$ & $1.0 \times 4$ & $\mathbf{P}$ & Gb,Ef & planned \\
\hline 19 & Raes06h & HSA12B-247 & B0809+74 & 17.12 .12 & 2.0 & $\mathbf{P}$ & Gb,Ef & planned \\
\hline 20 & Raes06k & HSA12B-247 & B1133+16 & 16.01 .13 & 2.0 & $\mathbf{P}$ & Gb,Ef & planned \\
\hline
\end{tabular}

Here are some estimates for the signal / noise ratio for these modes:

The full pulsar period is divided into $\mathrm{M}$ equal bins. $\mathrm{Z}$ is the Noise in a separate Bin. $\mathrm{S}(\mathrm{m})$ is the Signal in a separate Bin.

If we sum up all bins (no gate defined) we have:

$$
\frac{S}{N}=\frac{\sum_{m=1}^{M} S(m)}{\sqrt{\sum_{m=1}^{M} Z^{2}}}
$$

If we sum Bins with signal with either 0 or 1 weight for each bin (Simple Gate state), we obtain:

$$
\frac{S}{N}=\frac{\sum_{m=m 1}^{m 2} S(m)}{\sqrt{\sum_{m=m 1}^{m 2} Z^{2}}}=\frac{\sum_{m=1}^{M} S(m)}{\sqrt{\sum_{m=m 1}^{m 2} Z^{2}}}
$$

If each bin is weighted by the signal level in the current bin (for this mode we have to know the pulsar mean profile), we finally get:

$$
\frac{S}{N}=\frac{\sum_{m=m 1}^{m 2} S^{2}(m)}{\sqrt{\sum_{m=m 1}^{m 2}(S(m) Z)^{2}}}=\frac{\sum_{m=1}^{M} S^{2}(m)}{\sqrt{\sum_{m=m 1}^{m 2}(S(m) Z)^{2}}}
$$


The ASL Correlator can dedisperse data using the incoherent dedispersion algorithm. In this method for each frequency channel we have a corresponding (depending) time delay. The quality of dedispersion by this method depends on the number of frequency channels.

\section{Results}

Rafs01 experiment (Crab pulsar): correlations (visibilities) were found for selected strong giant pulses at both ground-ground and space-ground baselines. Observed GPs rate was 80 events per hour with SNR $>25(\mathrm{~S}>30000 \mathrm{Jy})$ with Evpatoria RT. The strongest GP was at a level $5^{*} 10^{5} \mathrm{Jy}$. RA-Earth distance was about $42000 \mathrm{~km}$, and the projected baseline was about $32000 \mathrm{~km}$.

Rafs 12 experiment (B0950+08 25.01.12): The correlation in a simple gating mode with the ASC correlator is completed. Notable visibilities were found on all baselines including space-ground ones. RA-Earth distance was about $290000 \mathrm{~km}$, and the baseline projection was about $175000 \mathrm{~km}$. A preliminary analysis of the shape of crosscorrelation functions indicates the presence of two scattering screens: one at a distance of about $40 \mathrm{pc}$ and the second one at a very close distance of $2 \mathrm{pc}$. The results of correlation on pulsar B0950+08 are presented in Fig. 1 as time-delay diagram. The correlation was performed with 512 channels and a $1 \mathrm{sec}$ integration time (about 4 periods); the gate window value was set to $15 \mathrm{~ms}$. We use a DM value equal to $2.969 \mathrm{pc} / \mathrm{cm}^{3}$.

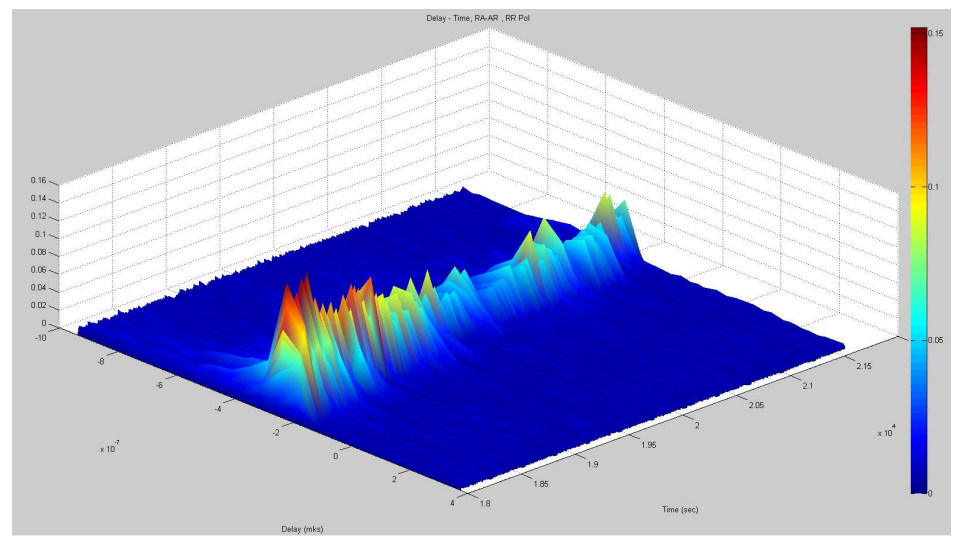

Figure 1.

Amplitude of visibility in time-delay coordinates for pulsar B0950+08 on baseline Radioastron-Arecibo

Rafs04 (a-b) experiment (Crab 02/05.03.12): Correlations (visibilities) were found in a $2 \times 30$-minutes intervals on strong giant pulses but only on ground-ground baselines; processing is being continued. RA-Earth distance was about $280000 \mathrm{~km}$ on March 2, and about $140000 \mathrm{~km}$ on March 6 (the baseline projections were about $145000 \mathrm{~km}$ and $120000 \mathrm{~km}$ respectively). In contrast to the Rafs01 case, we were unlucky with the strong GP rate: no events were observed 
with an SNR > 130 (150000 Jy) during the 8-hour observing period. Only 1-3 events are suitable to look for visibilities on space-ground baselines.

Raes07(a-b) experiment (Vela 10/18.05.12): ASC correlator made correlation with 1 second (10p) integration in a simple gating mode. Visibilities were found for both groundground and space-ground baselines. The scattering disk seems to be severely resolved. Namely, we detected fringes on the $100,000 \mathrm{~km}$ Radioastron-Tidbinbilla and Radioastron-Parkes baselines. The average normalized visibility was surprisingly $\langle\mathrm{V}\rangle=1.1 \%$, whereas a Gaussian model would predict a vanishingly small $<\mathrm{V}>\sim 10^{-150}$ [3].

Raes06d experiment (PSR1919+21 4.07.12): The correlation in a simple gating mode with the ASC correlator was completed. Notable visibilities were found on all baselines including space-ground ones. RA-Earth distance was about $90000 \mathrm{~km}$, and the projected baseline was about $70000 \mathrm{~km}$. Preliminary analysis of the fringe-rate versus radio frequency indicates the presence of two main scattered rays.

\section{References}

[1] Smirnova et al. 2008, ARep, 52, 736; 2006, A\&Ap, 453, 601

[2] Dennett-Thorpe \& de Bruyn 2002, Nature, 415, 57

[3] Gwinn et al. 2001, ApJ, 554, 1197; 2012, ApJ, 758, 6

[4] Narayan \& Goodman 1989, MNRAS 238, 963 ; MNRAS 238, 995

[5] Smirnova et al. 1996, ApJ, 462, 289

[6] Johnson et al. 2012, ApJ, 755, 179; ApJ 758, 8

[7] Popov et al. 2006, ARep, 50, 55

[8] Hankins et al. 2003, Nature, 422, 141

[9] Kondratiev et al. 2007, A\&AT, 286, 585 